\section{Workplace based assessments - confusion and misuse continues to be a problem}

Editor - We read with interest the study of trainee opinions towards workplace based assessment (WBA) implementation in medical training by Tailor et al (Clin Med 2014;14:510-6).

It is clear that much confusion remains as to the purpose of WBAs in the medical workplace, and that misuse of WBA tools is commonplace. ${ }^{1}$ It is also apparent that training bodies requiring ever-increasing numbers of WBAs to be completed only compounds the confusion between their formative and summative intent, which has again been highlighted in this study.

Education of both trainees and trainers as to the purpose of WBAs is lacking. Anecdotally, as surgical trainees in the $\mathrm{UK}$, we have never received formal training into the basis and formative intention of the WBA tools we have been required to utilise. One solution proposed to prevent this confusion is to 're-brand' WBAs as 'supervised learning events' (SLEs) - explicitly emphasising the learning intent. ${ }^{2}$ To date in the UK, only the Foundation Programme has formally introduced these into their curriculum - abolishing all tick-box elements of the tools, leaving only white-space boxes for trainer feedback and trainee reflection. ${ }^{3}$ The impact of this change on trainee, trainer, learning, engagement and perceptions will be appreciated in the near future and may encourage other training programmes to make this transition.

Another widely quoted factor underlying poor engagement with WBAs is 'time'; both that of trainers and trainees. Again, this study highlighted that the majority of trainers agreed that they found it difficult to complete the required number of assessments for their trainees due to time constraints. In this regard, it is notable that dental trainees appear to perceive
WBAs particularly favourably, almost in stark contrast to the perceptions of medical trainees that have been reported in the literature. ${ }^{4}$ Dental trainers have protected timetabled time for training, during which WBAs facilitate and direct learning and provision of feedback to their trainees. It seems idealistic to consider protected training time being introduced into consultant contracts, but this has certainly been raised as a potential solution to improving trainee engagement with WBAs in medicine.

AARON GOH

Clinical fellow in surgery, Cambridge University Hospital NHS

Trust, Cambridge, UK

JONATHAN MASSIE

Medical student, Cambridge University, School of Clinical Medicine, Cambridge, UK

JASON M ALI Academic clinical fellow, Department of Surgery University of Cambridge, Cambridge, UK

\section{References}

1 Ali JM. Getting lost in translation? Workplace based assessments in surgical training. Surgeon 2013;11:286-9.

2 General Medical Council. Learning and assessment in the clinical environment: the way forward. 2011. Available online at www. gmc-uk.org/Learning_and_assessment_in_the_clinical_ environment.pdf_45877621.pdf. [Accessed 18 October 2014].

3 Grieveson B, Kirton JA, Palmer NOA, Balmer MC. Evaluation of workplace based assessment tools in dental foundation training. Br Dent J 2011;211:E8.

4 UK Foundation Programme Office. The UK Foundation Programme Curriculum. Cardiff: UKFPO, 2014 